\title{
HISTORIA
}

\section{INVESTIGACIÓN DE LA ENFERMERÍA MILITAR VISTA A TRAVÉS DEL CINE: EL CASO DE PEARL hARBOR}

\section{Pedro Manuel Salas Iglesias}

Doctorando Universidad Alicante: Enfermería y Cultura de los Cuidados.

Licenciado Humanidades. Diplomado y Titulado Superior Enfermería

Enfermero Consejería Para la Igualdad y Bienestar Social. Junta Andalucía.

Delegación de Huelva

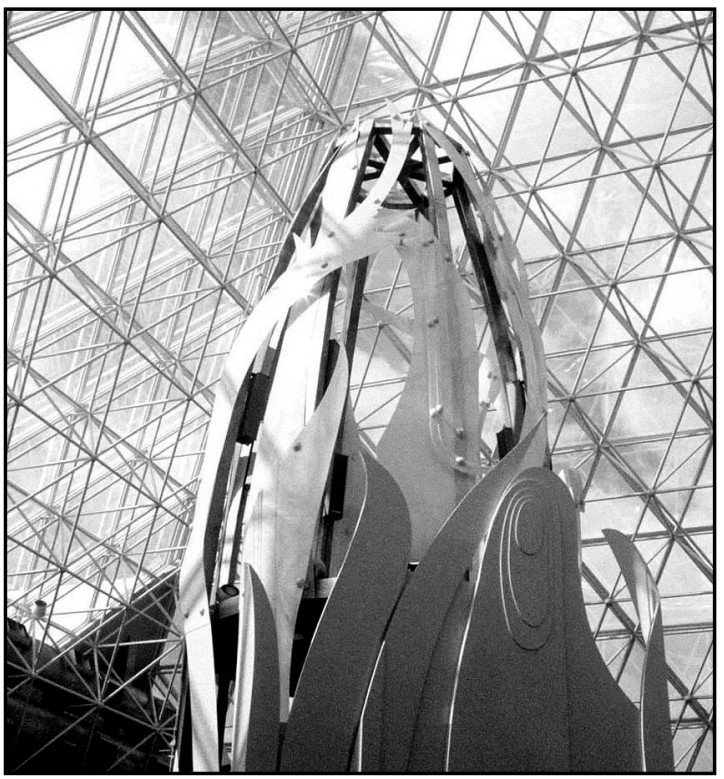

INVESTIGATION OF MILITARY NURSING THROUGH THE CINEMA: PEARL HARBOR

\section{ABSTRACT}

7 axis of this methodological-scientific investigation will be the historical moment of the U.S.A incursion in World War II, following the Japanese bombing to the main North American base in the Pacific, Pearl Harbor. The military cinema as mass media, becomes a useful tool for extracting the social image of nursing during the development of war conflicts, this image maintaining a historical-cultural relation with them. This film is articulated according to Florence Nightingale's nursing model.

Key words: Investigation, History, Anthropology of Cinema, Epistemology, Military Care, Pearl Harbor, World War II, Theories, Models, Florence Nightingale.

\section{RESUMEN}

71 eje vertebrador de la investigación metodológico-científica será el momento histórico de la incursión de los EEUU en la Segunda Guerra Mundial, tras el bombardeo japonés a la principal base norteamericana del pacífico en Pearl Harbor. El cine militar como medio de comunicación, es una buena herramienta para extraer la imagen social de la enfermería durante el desarrollo de los conflictos bélicos, manteniendo una relación histórico-cultural con los mismos. Esta película está articulada siguiendo el modelo de enfermería de Florence Nightingale.

Palabras clave: Investigación, Historia, Antropología del Cine, Epistemología, Cuidados Militares, Pearl Harbor, Segunda Guerra Mundial, Teorías, Modelos, Florence Nightingale.

\section{INTRODUCCIÓN}

Este estudio tiene la pretensión inicial de dar a conocer el papel y la imagen social, histórica y antropológica representada por la enfermería en la segunda guerra mundial. El elemento desencadenante que ha motivado dicha investigación, ha devenido por el transcurrir de los últimos acontecimientos belicistas como la guerra de Afganistán o 
la de Iraq, y que han hecho que la enfermería militar permanezca en auge y sea actual.

Otra de las causas justificativas habría que buscarla en la misma época histórica estudiada, pues se llegarán a formular los verdaderos precedentes de lo que devendrían los cuidados enfermeros militares de otras guerras mundiales posteriores. Por otro lado también, la fuente fílmica objeto de estudio, tiene el interés que precisamente la protagonista sea Enfermera Militar.

En la película merece la pena comentar, la existencia de una marcada ideología política propagandista imperialista de los EEUU, por la que el mismo presidente F.Rooselvet enunciaría respecto a la inclusión de su país en la guerra: "No se descuiden. El mundo está lleno de peligros y en cualquier momento la patria podría llamarlos a morir por la bandera" (lema falsacionista que bien recuerda al del presidente George Bush en su fatídica política bélica internacional).

La relación de la Enfermería en el film y con el momento histórico estudiado aparecerá desde el mismo momento en que los Estados Unidos declaren la guerra a Japón el 7 de Diciembre de 1941. El ataque fue dirigido por el comandante japonés Fuchida, con una duración de dos horas y llegando a destrozar cuatro viejos acorazados y otros buques de guerra.

Tras una batalla de cuatro años, la guerra finalizaría con el bombardeo nuclear de Hiroshima y Nagasaki, y una cuantía de más de 237.000 muertos.

De nuevo, la enfermería y los servicios de salud sufrieron las consecuencias de forma radical, pues el conflicto afectaría a cada hombre, mujer y niño de los países beligerantes.

La hipótesis previa para realizar el estudio y que se trata de demostrar es que la película estudiada constituye una buena fuente fílmica para configurar la historia y teoría de los cuidados militares en la segunda guerra mundial. Una segunda línea de investigación y subhipótesis pasa por intentar demostrar que en esta película los cuidados son reproducidos según el modelo de Florence Nightingale.

\section{OBJETIVOS:}

- Entre los objetivos generales a cubrir estarían:
Descubrir y analizar los cuidados militares en EEUU. Realizar una investigación interna y externa a esta fuente fílmica para estructurar los cuidados militares.

- Como objetivos específicos que se plantean están:

Aplicar la Heurística a la investigación de los cuidados militares en fuentes externas.

Aplicar la Hermenéutica al análisis interno , para: Describir y analizar los cuidados obteniendo resultados adscritos al modelo Nightingale. Demostrar que los cuidados militares son abordados de manera holística en esta película.

\section{ARGUMENTO DE LA PELÍCULA INVESTI- GADA:}

Rafe y Danny son dos amigos que han crecido juntos como hermanos, en una zona rural estadounidense y que han aprendido a volar en aviones fumigadores. Su amistad se ha prolongado a lo largo de los años convirtiéndose en pilotos de guerra aéreos. Rafe está enamorado de una enfermera militar (Evelyn) pero pronto es llamado a servir de manera voluntaria en la Fuerza aérea británica (RAF). Combatirá a los Nazis (como representante del ejército aliado norteamericano), mientras que Evelyn y Danny son transferidos al paradisíaco Pearl Harbor en Hawai. Rafe es dado por muerto, lo que hace que Danny y Evelyn se enamoren en medio del dolor y consuelo mutuo, dejándola embarazada. Pero Rafe sigue vivo y regresa a Pearl Harbor. Antes de decidir qué hacer ante esta dramática situación para los tres, Pearl Harbor es atacado por los japoneses. Como acto de venganza de los EEUU deciden enviar una misión suicida hacia Tokio, donde Rafe y Danny son reclutados para pilotar bombarderos. Desde el portaviones americano (USS Hornet) en el pacífico se acercan a 624 millas y lanzan sus bombas sobre las costas de Japón. Aunque las bombas que cayeron en la capital japonesa produjeron daños insignificantes, su repercusión moral fue inmediata, anunciando la resurrección del espíritu ofensivo de los EEUU y de nuevas derrotas.

El período final de la película y desenlace, acaba con la muerte de Danny que salva la vida a su amigo bloqueando las balas de las ametralladoras japonesas que intentaban capturarlos. Evelyn 
tras la guerra acabará el resto de sus días con Rafe, que criará al hijo de su mejor amigo y vivirán felices (Touchstone Pictures,Jerry Brucheimer Films (2001), http://www.alohacriticon.com/elcriticon /article989.html ) .

\section{FICHA TÉCNICA:}

En cuanto a la ficha técnica de la película tratada (http://www.notejode.net/peliculas/pearl\%20 harbor/harborhistoria.html, y en http://www.labutaca.net/films/4/pearlharbor.htm ), es la que se va a definir a continuación:

Dirección: Michael Bay. Ha dirigido otras películas entre las que destacan: Dos policías rebeldes, La Roca y Armageddon.

Guión: Randall Wallace.

Producción: Jerry Bruckheimer y Michael Bay. Montaje: Roger Barton, Mark Goldblatt, Chris Lebenzon y Steven Rosenblum

Interpretación: Ben Affleck (Rafe McCawley), Josh Hartnett (Danny Walker), Kate Beckinsale (Evelyn Johnson), Cuba Gooding Jr. (Doris 'Dorie' Miller), Dan Aykroyd (capitán Thurman), Tom Sizemore (Earl), William Lee Scott (Billy), Greg Zola (Anthony Winkle), Ewen Bremmer (Red), Alec Baldwin (Doolittle), James King (Betty), Catherine Kellner (Barbara), Jennifer Garner (Sandra), Michael Shannon (Theo), Jon Voight (presidente Roosevelt), Matthew Davis (Joe), Mako (almirante Yamamoto).

Música:Hans Zimmer.

Fotografia: John Schwartzman.

Género: Bélica.

País: EEUU.

Año: 2001.

\section{MARCO TEÓRICO Y ESTADO DE LA CUESTIÓN:}

Como partida de este estado de la cuestión cabe mencionar la referencia de otros autores que han investigado el cine como fuente científica empleándolo en la formulación de los postulados de la histórica y antropológica: Shapiro JF (1998), Alonso Barahona,F (1992), (Sola A, Selva M (1991)), Leguineche M.(2001), Choza J. (2002), Fuentes Goyanes E.( 1982). Existen estudios internacionales que giran en torno a la figura de enfer- mería a través del cine: Kalish P(1981), Newby M (1989), Hirata, MC. (2001), Lewes H (1995), y en territorio nacional: (Siles,González J, Cibanal Juan.L, García Hernández E, Gallardo Frías Y, Lillo Crespo M (1998)), Salas Iglesias,PM (2000), (Andina Díaz,E ,Bonet Manso,P (2004)). El tema de los cuidados militares ha suscitado investigaciones de la sanidad militar (Martinez Asencio , Palacio Gavin, (2003 )y de la historia de la enfermería militar en España (González Yánez, 2003).

Como marco teórico es importante en primer lugar establecer un análisis antropológico del espíritu histórico y cultural del hombre japonés que existía durante la guerra, y para ello hay que partir del momento crucial histórico fundamentado en el afán de Japón por dominar el Pacífico (incorporándose de esta manera al club de las grandes potencias mundiales). Hay elementos antropológicos en torno al carácter agresivo del banzai japonés, con un espíritu de orgullo de raza (Whitney J.,Leguineche M.). El éxito momentáneo de Pearl Harbor conduce al harakiri de muchos almirantes y generales norteamericanos. Se establece un etnocentrismo cultural con el hombre japonés, ya que se compara su cultura con la americana(por parte de algunos generales )y es considerada inferior al describirlos como: pilotos miopes, de dientes podridos por la caries, gente incapaz de poner en pie una potente maquinaria de guerra, de estatura pequeña y poco corpulenta, empleo de adjetivos diminutivos ("los japos"( de japonés)llegándose a alcanzar ideas de índole racista). En el cine esta infravaloración antropológica se verá reflejada en películas como El Puente sobre el Río Kwai (1957), que muestra fenomenológicamente la incapacidad de unos militares japoneses para construir un puente. En cambio, la visión que tiene la cultura japonesa sobre el hombre occidental se encuadra en un fomento de ideas de tipo despreciativas hacia el pueblo americano: materialista, corrupto, aislacionista, desdeñoso de la política de defensa, decadente....

Cuando se enfoca este marco teórico en los cuidados militares es notable prefijar como referencia, la situación que la enfermería ha establecido con el potencial configurador de la paradoja en relación con los conflictos bélicos(Siles,1999). Se pueden llegar a distinguir los siguientes elementos: 1. 
Conocimiento de técnicas y cuidado de heridas, 2. Conocimiento de procedimientos en cuidados a enfermos, 3. Incidencia del apoyo sanitario en la eficacia del ejército, 4. La relación entre el factor político ideológico y la forma de organizar y desarrollar los servicios sociosanitarios, 5. La guerra como caudal de significados e interpretaciones de experiencias personales.

Con respecto a la enfermería militar en EEUU se hace necesario definir la etapa de la Historia de los cuidados militares durante la Segunda Guerra Mundial (Donahue,1993).

En Julio de 1940 se constituye el Consejo de Enfermería para la Defensa Nacional. Su estructura constitucional, se integra por las seis organizaciones nacionales de Enfermería (ANA, NLNE,NOPHN, ACSN,NACGN, AAIN), los servicios de enfermería federales y representantes de organizaciones como la American Hospital Association. En 1942 con la declaración de guerra el nombre será el de Consejo Nacional de Enfermería para el Servicio de Guerra, del que Stella Goostray es su nueva jefa.

Tras la creación de la Ley Pública $n^{\circ} 74$, LXXVIII del Congreso (conocida como Acta Bolton), se creó el Cuerpo de Enfermeras Cadetes de los EEUU. El programa formativo abarcaba 30 meses; las cuotas, estudios y uniforme eran gratuitos, existía una paga mensual para las estudiantes de las escuelas de Enfermería reconocidas, así como becas de trabajo de postgraduación (enfermeras graduadas).

Por otra parte la Guerra de Crimea y el papel desempeñado por Florence Nightingale, había revolucionado el concepto y el trabajo desarrollado por las enfermeras militares. De hecho, en la Segunda Guerra Mundial, las enfermeras (que serían una parte integrante de la estructura militar), estaban acostumbradas a la organización, habían adquirido un conocimiento experimental (a través de sus propias experiencias) y su preparación previa les facultaba para poder cubrir las necesidades de una guerra moderna de este tipo. Encontraríamos enfermeras militares en cerca de cincuenta países diseminados en los principales puntos de enfrentamiento bélico. Los lugares habituales en los que vivían y trabajaban abarcaban desde las instalaciones del Ejército y la Marina, hoteles y estructuras adaptadas, barracas de acuartelamiento, tiendas-hospital, chozas de material prefabricado, etc.

La Enfermería comenzará una nueva faceta en la II Guerra Mundial al ejercer sus funciones durante el vuelo militar. Colaborarán en el transporte aéreo como medio de evacuación de los heridos. Los cuidados a los heridos se realizaban en los lugares donde eran colocados en literas de tres a ambos lados de los aviones de carga ( a modo de aviones ambulancia). Para acceder a la escuela de enfermería de vuelo, se precisaba previamente formar parte del Cuerpo de Enfermeras del Ejército, continuando con un período mínimo de seis meses en un hospital de la Unidad de las Fuerzas Aéreas. Tras cumplir con estos requisitos se podía acceder a la escuela de enfermería de vuelo.

\section{MATERIAL Y MÉTODOS:}

Se trata de un estudio descriptivo transversal de corte histórico-antropológico para los cuidados enfermeros. Se realizará una investigación bifásica cualitativa empleando en la instrumentación de procesamiento de datos, las técnicas de la Heurística (análisis externo a partir de las bases de datos de la red internet) y la Hermenéutica (análisis interno derivado de la propia película).

Los materiales consultados se han investigado en ocho bases de datos de la red internet: Cuiden, Cuidatge, BDIE, TESEO, ISBN, Medline, Biblioteca del Congreso de los Estados Unidos y Corhane. Se han introducido diferentes descriptores que han sido traducidos al inglés para las fuentes internacionales. Entre ellos se encuentran los siguientes: "enfermería militar", "cine", "Pearl Harbor", "segunda guerra mundial","antropología del cine", "guerras", "cuidados militares", "historia de la enfermería militar". Se desecharon los resultados para: la categoría "guerra" por su alto nivel de inespecifidad y poca pertinencia a los cuidados militares, así como con el tratamiento dado para la categoría "historia de la enfermería militar" (debido a los escasos resultados representativos). En la investigación de "cine" se añadió la categorización: "movies nursing" y "cinema nursing" en las bases internacionales.

Para el tratamiento del análisis cuantitativo se ha recurrido al programa informático de cálculo 
Excel de Windows XP, así como para su representación gráfica.

La interpretación Heurística ha sido estructurada según la propuesta investigativa del Dr. J.Siles (catedrático de Enfermería y Director de la Asociación de Historia y Antropología de los Cuidados). Por otro lado se han seguido las directrices metodológicas heurísticas marcadas por la investigación de la enfermería basada en la evidencia, en la búsqueda y obtención de resultados significativos.

Para el análisis Hermenéutico se ha seguido las directrices de la adscripción al paradigma hermenéutico de Habermas J., aplicados a una fuente fílmica.

\section{RESULTADOS:}

En cuanto a los resultados cuantitativos son los obtenidos en gráfica 1.

De la investigación heurística respecto al ataque a Pearl Harbor en el cine, se descubre que la temática sobre la que versa la película no es original. Así en 1953, Fred Zineman Rueda "De Aquí a la Eternidad", donde se ve reflejado el ambiente confiado que se vivía en la base americana en vísperas de la guerra. Años más tarde en 1970 otra película: "Tora, Tora, Tora", dirigida por Richard Fleischer muestra a los japoneses convertidos en gran potencia económica, con una visión de no ser ya malos

(en http:://www.teachwithmovies.org/guides/tora-toratora.html).

De los resultados heurísticos a nivel de historiadores revisionistas se arrojan los descritos a continuación: la existencia de una serie de fallos y negligencias por parte de los americanos para descubrir y evitar el desastre que se avecinaba en Pearl Harbor. Entre ellos destacan: responsables de la seguridad que vieron la aproximación en pantalla de radar-móvil al norte de la isla de Oahu de al menos cincuenta aviones, confundiéndolo con aviones americanos B-17 que llegaban desde California, y sin tomar medidas de seguridad declaran tan sólo a sus soldados "tranquilos chicos,tomaos un té bien cargado" (Leguineche M.). O también la posición en hilera, de dos en dos de los buques de guerra norteamericanos en la Avenida de los Acorazados, baterías desarmadas por temor a los sabotajes de una quinta columna japonesa, aparatos de las bases aéreas que también fueron vaciados de combustible y colocados juntos para que fuese más fácil vigilarlos. Se incluye además el gran descuido de esa quinta columna japonesa en las islas Hawai, entre la que se mueven con total facilidad decenas de espías que proporcionaban cuantas informaciones y fotografías aéreas demandaban los generales de Tokio.

Existen resultados heurísticos también encontrados sobre algunos fallos desde el área japonesa: los tres portaaviones que Washington tenía en el Pacífico (Enterprise, Lexington y Saratoga) salieron indemnes del ataque japonés (porque en el momento del ataque se encontraban en otras aguas), el bombardeo sorpresa por aparatoso que fuera sirvió para poco, ya que la orden dada por el almirante japonés Naguno de retirarse del espacio aéreo de Pearl Harbor (antes de un tercer golpe), no consiguió destruir los depósitos de combustible, todos los talleres, almacenes, muelles, decenas de barcos auxiliares, etc. En pocos meses la base al quedar poco dañada volvió a funcionar al ciento por ciento, reflotándose parte de los barcos dañados y permitiendo reiniciar el ataque contra Japón.

Por consiguiente, se puede deducir que de lo que Tokio imaginó como un golpe decisivo a la moral americana, se transformó en la voluntad común de vengar la humillación de Pear Harborl.

De la segunda fase de la investigación y como resultados Hermenéuticos se han encontrado los que se exponen a continuación:

La enfermería militar de los años cuarenta, es utilizada como mecanismo humano que no cumple en el film un objetivo claro de tipo vocacional al alistarse al ejército para la prestación de cuidados, sino que se solapa por perseguir una doble meta: por un lado el cumplimiento del patriotismo y por otro como base para entablar relaciones amorosas con los soldados. Si bien el hilo argumental, gira en torno precisamente al drama amoroso entre un oficial piloto y su novia enfermera militar.

El lugar donde se desempeñan las funciones de enfermera militar son recogidos en el film de forma clara:

1. Centros de Reconocimiento Militar. Ubicados fuera del frente, en grandes ciudades, cercano a bases militares, aeródromos, etc. Las enfer- 
meras y médicos realizan exámenes y reconocimientos médicos para obtener el certificado médico que les faculta para poder pilotar aviones, dar la autorización sanitaria para ir a luchar al frente ,etc. No es sólo suficiente demostrar conocimientos militares en Academias Militares, aprobando exámenes teóricos de distintas materias sino de tener el Gobierno una garantía a efectos de salud, de que están sanos. En estos Centros la Enfermería suele ser femenina , uniformada de blanco, con falda, cofia, delantales y capa de color negro (empleada cuando salen a la calle). Las funciones que desempeñan van desde la administración de inyectables, inoculación de vacunas, revisiones oculares junto con los médicos, toman controles habituales: presión arterial, temperatura, pulso, así como prestación de Cuidados de Enfermería ante situaciones de urgencias ejemplificados en la película en dos momentos: (a). Reacción anafiláctica que sufre Rafe (protagonizado por Ben Haffleck) tras la administración de una vacuna (inyectada por error por cambio de historial médico correspondiente a otro enfermo). (b). Hemorragia nasal traumática producida al descorchar champán francés e impactarle en la nariz a Rafe (novio), cuando están junto a la enfermera Evelyn, ésta intenta cohibir la hemorragia, se le practica taponamientos nasal, y se le aplica hielo local, etc.

2. Hospitales militares base: Ubicados cerca de bases militares, integrados en el casco urbano. Atienden heridos leves que precisan larga recuperación, fracturas, cura por quemaduras de marineros, etc. En la película es el Hospital de la Base de la Marina de Pearl Harbor y será durante el ataque japonés Centro de Referencia Principal Sanitario para todas las urgencias, heridos, etc. de la isla Ohau. El uniforme de las enfermeras en estos Hospitales es de color blanco, con faldas y cofias. La estructura física del Hospital consta de una gran nave muy larga con múltiples ventanales, muy amplios, que permite una constante ventilación del lugar, con cortinas poco tupidas para no impedir el paso de la luz, cercana a estas ventanas se colocan las camas de tubo, una al lado de la otra. En las paredes de enfrente se sitúan las estanterías de material, también de color blanco.

Durante la situación de bombardeo japonés el hospital sufre una transformación: alejan a los enfermos de las ventanas y los cubren con colcho- nes para protegerlos de los aviones japoneses. Para poder prestar todos los cuidados de urgencia y emergencia necesarios se llegan a ocupara todas las dependencias y salas del hospital, incluyendo mesas de cocina.

Las enfermeras serán las encargadas de valorar tras delegación del servicio médico, ya que las reservas de morfina del Hospital se están acabando, a los enfermos que han recibido poca morfina (realiza marcas en la frente de los enfermos, empleando para ello su propia barra de labios). Debido a que se ven saturados por el gran número de heridos que siguen llegando al hospital, necesitan organizarse, para que sólo entren los que puedan salvarse. Dicha función (de lo que sería en la actualidad la función de Triage) de valoración y clasificación es encargada a la enfermera Evelyn, marcando en la frente de nuevo con lo primero que tienen a mano, su barra de labios con distintas letras indicativas de su estado de salud $(\mathrm{C}, \mathrm{F}, \ldots .$.$) .$ Dentro del hospital la enfermera llega a realizar todas las funciones de su campo profesional para salvar la situación bélica, siendo totalmente necesario, salvar vidas. Así se llegan a taponar arterias carótidas con compresión digital, por la falta de medios materiales se recurren para hacer torniquetes las propias medias de las enfermeras, en el hospital precisan de transfusiones urgentes de sangre, siendo los recipientes donde se recogen botellas de cristal de CocaCola Se prima sobretodo el estado de salud y las posibilidades de vida que aún tengan dichos heridos.

Como resultados hermenéuticos con respecto a la metodología enfermera que aparece en la película, es notable la influencia del modelo de Florence Nightingale (Wesley) y su teoría del entorno (excepto en situaciones de bombardeo franco en zonas donde se ubiquen los hospitales base). De acuerdo con Nightingale, las influencias y condiciones externas poco saludables durante la guerra (falta de higiene en campamentos) pueden prevenirse, suprimirse, o contribuir a la enfermedad y muerte. Es objetivo central del modelo ayudar al paciente a mantener su propia vitalidad, mientras satisfacía sus necesidades gracias al control del entorno. Define cinco componentes fundamentales que integran un entorno positivo o saludable: ventilación adecuada, luz adecuada, calor suficiente, control de los efluvios y control del ruido. 
Nightingale consideró tres tipos de entorno: físico, psicológico y social. En la película se verán reflejados al registrarse las condiciones del entorno físico y psicológico presentes en los hospitales de base de Pearl Harbor: buena ventilación con amplios ventanales, por donde entra mucha luz, el medio ambiente es apropiado con poco frío, (isla Oahu en Hawai y con mucha tranquilidad cuando no existen bombardeos, de hecho se practican deportes náuticos variados como el incipiente surf), realización de control de efluvios, buenas medidas higiénicas, todas las paredes y suelos son blancos, lo que relaja el sentido de la vista dando sensación de mayor asepsia y limpieza. Las enfermeras que desempeñan allí su labor mantienen buenos niveles de comunicación con los enfermos.

De acuerdo con Nightingale en los hospitales del film, la enfermería influirá sobre el entorno de la persona de forma que le ayude a restaurar la salud, y aunque la teórica no describa específicamente el proceso de enfermería, su teoría lo apoya (la enfermera Evelyn llegará a realizar todo un proceso de enfermería con las cinco etapas: valoración, diagnóstico, planificación, ejecución y evaluación cuando estalla el bombardeo nipón y hay que atender a la gran cantidad de heridos que se desencadenan.).La obra fílmica recoge también los cuatro conceptos del metaparadigma de la enfermería incluidos en la teoría de Nightingale: persona, entorno, salud, enfermería.

\section{DISCUSIÓN Y CONCLUSIONES:}

La investigación realizada consigue demostrar la hipótesis iniciadora planteada. Se puede concluir que un medio de comunicación como es el cine puede proporcionar información sobre la imagen social de la enfermera a nivel de la historia y la antropología en la segunda guerra mundial. Existen ciertos matices criticables que desvirtúan el ejercicio serio profesional, al usar la disciplina como mecanismo humano para ligar y alcanzar el amor y desvinculándola de la visión historiográfica de este período de la historia de los cuidados. Sin embargo esta situación no es generalizable, alistándose al ejército por causas reales del deber de patriotismo al país que represente, y practicando una enfermería de ayuda con marcado acento sociohumanitario y serio cumplimiento del deber.
En cuanto al número de investigaciones realizadas de la enfermería en el cine a pesar de la aportación documental a la historia y antropología de los cuidados (así como a su objeto metodológico de la Cultura de los Cuidados) existen pocos estudios aún a nivel nacional, por lo que se convierte en una futura fuente de investigación por explorar. La Segunda Guerra Mundial y el ataque de Pearl Harbor fue una gran prueba que superamos pero que jamás volveríamos a olvidar.

Los distintos factores de índole negativo que intervinieron en esta guerra como el término de la vida (muertos) así como la interrupción de los procesos de salud (heridas, lesiones, enfermedades epidémicas, etc.) han permitido el avance en el conocimiento experimental y empírico-científico en la prestación de cuidados bélicos, la macrohistoria y antropología de los cuidados, así como su ampliación también en la posible interconexión de Enfermería con otras disciplinas.

Concluimos el estudio con el espíritu de animación al colectivo enfermero para que investigue y podamos conseguir entre todos, un avance en el conocimiento epistemológico y paradigmático de los cuidados, como instrumento consolidado que permita actuar ante las nuevas necesidades sociológicas, antropológicas, psicológicas y culturales surgidas en esta nueva era de los medios de comunicación.

Intentemos vivir en tiempos de paz armónicamente, asentando las bases para la construcción de la enfermería como una ciencia epistemológica de los cuidados y huyamos de las situaciones conflictivas porque la guerra es la mayor plaga que puede afligir a la humanidad. (Martín Lutero, 14831546).

\section{BIBLIOGRAFÍA:}

- Alonso Barahona,F (1992). Antropología del cine. Centro de Investigaciones Literarias Españolas e Hispanoamericanas. Barcelona.

- Andina Díaz E, Bonet Manso P (2004).La enfermería en "Hablé con ella".Index Enfermería.Granada, (44-45):67-71.

- Choza J. (2002).La antropología en el cine occidental. Madrid:Ed.del Laberinto.

- Donahue,M.P.(1993).Historia de la Enfermería. Doyma. España:410-420.

- El Criticón-Pearl Harbor de Michael Bay: http://www.alohacriticon.com/elcriticon/article989.html. (Marzo 2004).

- Fuentes Goyanes,E.( 1982). El cine científico una actividad académica normal. Rev. Psicología Universitas Tarraconensis. V-IV (1):31-38. 
- González Yánez,J.(2003).Historia de la enfermería militar española. Santa Cruz de Tenerife. Canarias.Ed. GONZÁLEZ YANES,JERÓNIMO.

- Habermas,J.( 1988).La lógica de las Ciencias Sociales.Madrid. Tecnos.

- Hirata, MC. (2001). O cuidado e a força do cinema na criaçao do mito da enfermira heroína na II Guerra Mundial. Alcalá de Guadaira, Sevilla.

- Kalish P. (1981).When nurses were national heroines:images of nursing in America film. 1942-1945.Nursing Forum, 20 (1),14-6.

- La butaca-Pearl Harbor: http://www.labutaca.net/films/4/ pearlharbor.htm (Marzo 2004).

- Leguineche,M.(2001).Pearl Harbor.El Cebo. Rev. La Aventura de la Historia. Alianza Ediciones.Madrid: 30-39.

- Lewes H (1995). 20th Century Fox: Nurses have fascinated film makers ever since cinema began a century ago. Nursing Times, 91 (50), 36-7.

- Martínez Asencio S, Palacio Gavin G(2003). Enfermería del cuerpo de sanidad militar. Zaragoza.Ed.Master- Distancia.

- Newby M (1989).Nursing at the movies. Nursing Standard, 3,13 .

- Pearl Harbor una película histórica: http://www.notejode.net /peliculas/pearl\%20harbor/harborhistoria.html (Marzo 2004).
- Pearl Harbor: http://www.pearlharbor.com. (Marzo 2004).

- Salas Iglesias,PM (2000).Investigación cualitativa sobre "Vértigo" de Alfred Hitchcok desde una perspectiva humanístico enfermera. Documentos Enfermería. IV (15),15-17.

- Shapiro JF. (1998). Atomic bomb cinema. Spring 17 (1): 12648.

- Siles González,J. (1999).Historia de la Enfermería. Aguaclara. Alicante.

- Siles,González J, Cibanal Juan.L, García Hernández E (et al)(1998). La Enfermería en el Cine. Imagen durante la Guerra Civil Española. Rol de Enfermería, XXI (244), 25-31. Barcelona.

- Sola A., Selva M (1991). El cine: imagen y conocimiento. Infancia y Sociedad. V(7):101-108.

- Tora-Tora-Tora:www.teachwithmovies.org/guides/tora-toratora.html

- Touchstone Pictures,Jerry Brucheimer Films (2001). Pearl Harbor. USA.

- Wesley,R.L(1997). Teorías y Modelos de Enfermería. McGraw-Hill.Interamericana,México:5-14.

- Willmott, H.P. (2001). Pearl Harbor. Londres, Cassell \& Co.

- Whitney,J.(1973). El Imperio Japonés. Rev. Historia Universal. Siglo XXI. Madrid.

Relación de las investigaciones en bases de datos

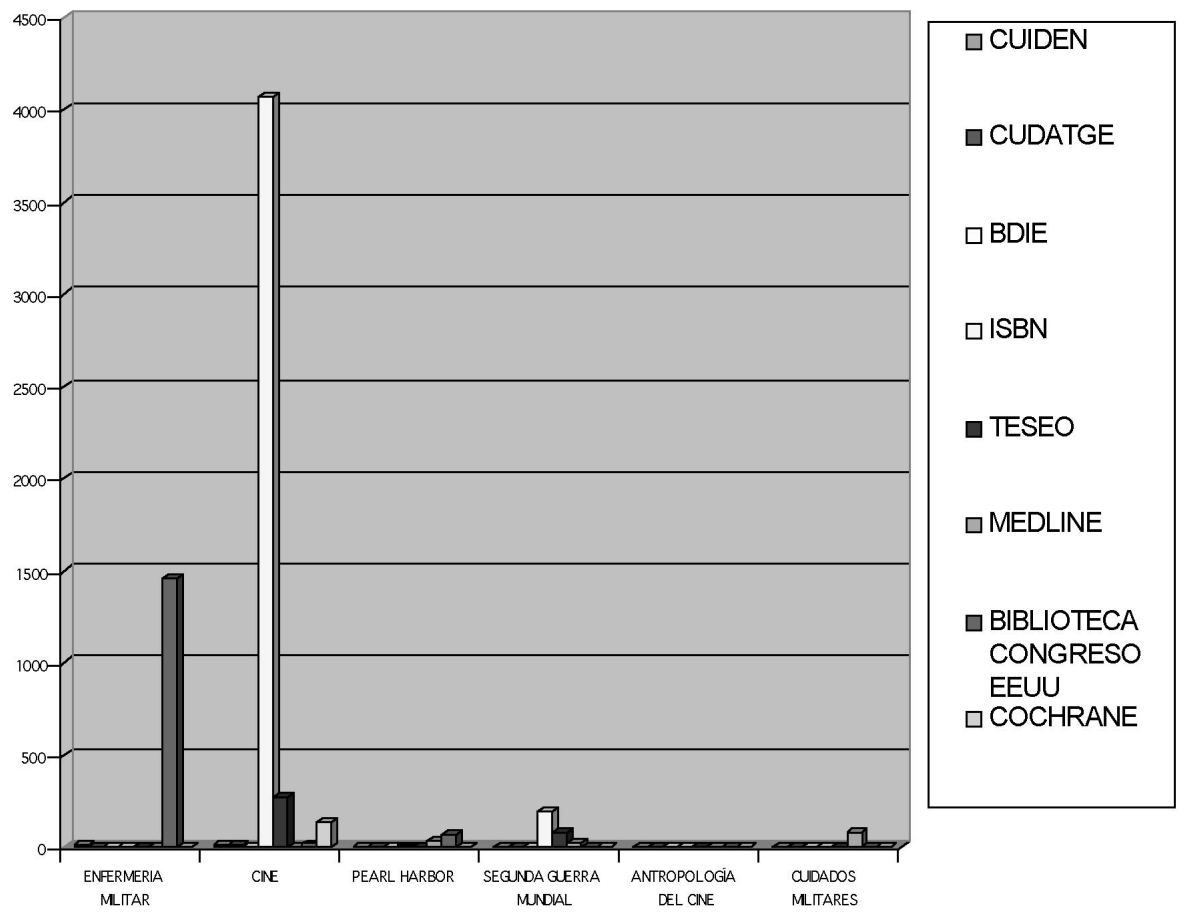

